\title{
Consumers' Willingness to Pay for Privacy Services
}

\author{
Frauke Mattison Thompson and Kirk Plangger
}

\begin{abstract}
To alleviate consumer privacy concerns, governments have introduced privacy regulations to manage excessive consumer privacy intrusion by organizations (Solove 2008; Bennett 2008). Yet research shows that these regulations are not sufficient in reducing consumer privacy fears as privacy concerns vary in magnitude from consumer to consumer (Mattison Thompson 2007) and the law can only protect against privacy intrusion on a uniform level. Despite the extensive body of research investigating the variance in consumer privacy concerns within the context of legislative boundaries (e.g., Milne et al. 2004; Milne et al. 2006; Shilton 2009; King and Raja 2012; King and Jessen 2010), little has been done to understand whether consumers would be willing to pay for additional privacy services offered by organizations to protect their privacy above and beyond the protection the law provides. Since consumers vary in their privacy needs, one solution that firms could adopt to reduce their customers' privacy concern is to offer privacy as an individualized, personalized service or "Privacy Services" that cater to the higher privacy needs of some customers. Privacy services involve a customer paying an additional fee to receive privacy protection above what is offered to other customers, thus potentially mitigating the customers' privacy concern for transactions they have with that firm.

In this study, we explore how consumer privacy concern impacts consumers' willingness to pay for "Privacy Services," as well as the moderating effect of consumer trust on this relationship. We present empirical evidence from over 16,000 consumers in 20 countries. We contribute to the consumer privacy literature by showing that consumer privacy concern influences consumers' willingness to pay for "Privacy Services," and that consumer trust moderates this relationship.
\end{abstract}

References available upon request.

F.M. Thompson $(\bowtie) \cdot$ K. Plangger

King's College London, London, UK

e-mail: frauke.mattison_thompson@kcl.ac.uk; kirk.plangger@kcl.ac.uk 